Mon. Not. R. Astron. Soc. 000,1-?? () Printed 31 July $2018 \quad$ (MN LATEX style file v2.2)

\title{
Standing shocks in magnetized advection accretion flows onto a rotating black hole
}

\author{
Santabrata Das*, Biplob Sarkar \\ Indian Institute of Technology Guwahati, Guwahati, 781039, India.
}

Accepted . Received ; in original form

\begin{abstract}
We present the global structure of magnetized advective accretion flow around the rotating black holes in presence of dissipation. By considering accretion flow to be threaded by toroidal magnetic fields and by assuming synchrotron radiative mechanism to be the dominant cooling process, we obtain global transonic accretion solutions in terms of dissipation parameters, such as viscosity $\left(\alpha_{B}\right)$, accretion rate $(\dot{m})$ and plasma- $\beta$, respectively. In the rotating magnetized accretion flow, centrifugal barrier is developed in the nearby region of the black hole that triggers the discontinuous shock transition in the flow variables. Evidently, the shock properties and the dynamics of the post-shock flow (hereafter post-shock corona (PSC)) are being governed by the flow parameters. We study the role of dissipation parameters in the formation of standing shock wave and find that global shocked accretion solutions exist both in gas pressure dominated flows and in magnetic pressure dominated flows. In addition, we observe that standing shock continues to form around the rapidly rotating black holes as well. We identify the range of dissipation parameters that permits shocked accretion solutions and find that standing shocks continue to form even in presence of high dissipation limit, although the likelihood of shock formation diminishes with the increase of dissipation. Further, we compute the critical accretion rate $\left(\dot{m}^{\text {cri }}\right)$ that admits shock and observe that standing shock exists in a magnetically dominated accretion flow when the accretion rate lies in general in the sub-Eddington domain. At the end, we calculate the maximum dissipated energy that may be escaped from the PSC and indicate its possible implication in the astrophysical context.
\end{abstract}

Key words: accretion, accretion discs, magnetic field, black hole physics, magnetohydrodynamics (MHD), shock waves

\section{INTRODUCTION}

Magnetic fields are in general considered to be indispensable in the astrophysical environment and therefore, their presence in the accretion disc is by all means inevitable (Balbus \& Hawlev 1998). In a magnetized accretion disc, magnetic fields play an important role in guiding the infalling matter around black holes. Meanwhile, Blandford \& Pavne (1982) revealed that when a Keplerian disc is threaded by large scale magnetic fields, angular momentum can be removed through the torque exerted by the magnetic fields. Similarly, the large scale poloidal magnetic fields anchored in the surrounding accretion disc are indeed capable of transferring energy and angular momentum and also instigate the generation of powerful magnetic jets (Blandford \& Znajek 1977; Komissarov \& McKinney 2007).

* E-mail: sbdas@iitg.ernet.in; biplob@iitg.ernet.in
Further, Balbus \& Hawley (1991, 1998) showed that the accretion disc becomes unstable in presence of differential rotation when the accreting plasma is threaded by weak vertical magnetic fields. This instability causes the turbulence leading to the efficient angular momentum transport as well as energy dissipation that enables the accretion possible.

In the modeling of the standard advection-dominated accretion flows around black holes, Narayan \& Yi (1995) considered the magnetic fields which are stochastic in nature. However, since the flow experiences differential rotation while accreting onto a black hole, the magnetic fields present in the disc are expected to be structured in reality and the large scale fields seem to be dominated by its toroidal component. This consideration in general holds irrespective to the initial configuration of the fields (i.e., toroidal or poloidal). Furthermore, the existence of toroidal magnetic field has been observationally confirmed in the exterior regions of the discs of young stellar objects Aitken et al. 
1993; Wright, Aitken, \& Smith 1993) as well as in the Galactic center (Chuss et al. 2003; Novak et al. 2003). Meanwhile, significant efforts were given to examine the accretion disc properties around black holes including toroidal magnetic fields (Akizuki \& Fukue 2006; Khesali \& Faghei 2008, 2009; Mosallanezhad. Abbassi. \& Beiranvand 2014; Mosallanezhad. Bu. \& Yuan 2016; Oda et al. 2007, 2010, 2012; Samadi. Abbassi. \& Khaiavi 2014; Sarkar \& Das 2015, 2016; Sarkar. Das. \& Mandal 2018; Sarkar \& Das 2018). Following the above cognizance, in the present work, we consider the accretion flow to be threaded by toroidal magnetic field lines as well.

Further, while developing the present formalism, we consider rotating matter that experiences centrifugal repulsion as it accretes towards the black hole and due to this, infalling matter is being piled up in the vicinity of the black hole. In reality, such accumulation of matter can not be continued indefinitely and ultimately, at its limit, the centrifugal barrier triggers the discontinuous transition of the flow variables which is commonly called as shock transition. It may be noted that the global accretion solutions including shock waves are potentially favored as it owns large amount of entropy Becker \& Kazanas 2001). In the theoretical front, the shock induced global accretion solution around black hole and its implications are extensively studied by the numerous groups of workers (Fukue 1987; Chakrabarti 1989, 1996b; Lu, Gu, \& Yuan 1999; Gu \& Lu 2001; Das et al. 2001b; Gu \& Lu 2004; Fukumura \& Tsuruta 2004; Chakrabarti \& Das 2004; Mondal \& Chakrabarti 2006; Das 2007; Becker. Das. \& Le 2008; Das, Becker. \& Le 2009; Das. Chakrabarti, \& Mondal 2010; Sarkar \& Das 2015; Aktar. Das, \& Nandi 2015; Sarkar \& Das 2016; Aktar et al. 2017; Sarkar \& Das 2018; Sarkar. Das, \& Mandal 2018). In addition, the existence of shock in accretion flow is also examined numerically considering hydrodynamics (Chakrabarti \& Molteni 1993; $\quad$ Molteni. Lanzafame. \& Chakrabarti 1994; Rvu. Chakrabarti. \& Molteni 1997; Okuda 2014; Okuda \& Das 2015; Suková \& Janiuk 2015; Suková. Charzvński, \& Janiuk 2017) as well as magnetohydrodynamic (MHD) environment (Nishikawa et al. 2005; Takahashi et al. 2006; Fukumura, Takahashi, \& Tsuruta 2007; Fukumura et al. 2016).

Motivated with the above studies, in this work, we examine the magnetically supported accretion flow around rotating black hole that possesses standing shock. While doing this, we assume that the characteristics of the magnetic pressure is synoptic to the gas pressure and their combined effects therefore supports the vertical structure of the infalling matter against the gravitational pull. Moreover, recalling the success of the seminal $\alpha$-viscosity prescription (Shakura \& Sunvaev 1973), we consider the Maxwell stress to be proportional to the total pressure (Machida, Nakamura, \& Matsumoto 2006) that evidently demonstrates that the outward transport of angular momentum would certainly be enhanced as the magnetic activity inside the disc is increased. Furthermore, we consider the heating of the flow to be regulated by the magnetic energy dissipation mechanism while the inflowing matter is being cooled via synchrotron emission process Chattopadhyay \& Chakrabarti 2000; Das 2007; Sarkar. Das. \& Mandal 2018). In addition, for simplicity, we adopt a pseudo potential introduced by Chakrabarti \& Mondal (2006) that successfully mimics the space-time geometry around the rotating black hole having spin $a_{k} \lesssim 0.8$. Considering all these, we self-consistently solve all the governing equations that describe the magnetized accretion flow around rotating black hole and obtain the global accretion solutions including shock waves. We study the properties of standing shock waves in terms of flow parameters and observe that shock formation takes place for an ample range of parameters both around weakly rotating $\left(a_{k} \rightarrow 0\right)$ as well as rapidly rotating black holes $\left(a_{k} \sim 0.8\right)$. We also calculate the critical accretion rate $\left(\dot{m}^{\text {cri }}\right)$ for standing shocks in magnetized accretion flow. It may be noted that $\dot{m}^{\text {cri }}$ does not bear any universal value, rather it is largely dependent on the inflow parameters. We continue our study considering the fact that standing accretion shocks are dissipative by nature and calculate the maximum energy that can be extracted from the PSC. In reality, this available energy could be utilized in powering the jets (Sarkar \& Das 2016, reference therein) as they seem to originate from PSC regions Aktar et al. 2017, reference therein).

We organize the paper as follows. In $\S 2$, we write the model equations and carry out the analysis of transonic conditions. In $\S 3$, we display our results where shocked accretion solutions for magnetized flow and its properties are discussed. Moreover, we determine the critical inflow parameters for standing shock as well. We further study the characteristics of dissipative standing shock. Finally, in $\S 4$, concluding remarks are presented.

\section{ACCRETION FLOW MODEL}

To take into consideration of the magnetic fields structure in an accretion disc, we rely on the numerical simulation results of global and local MHD accretion flow around black hole. These simulations have revealed that magnetic fields inside the accretion disc are turbulent and primarily dominated by the azimuthal component (Hirose. Krolik. \& Stone 2006; $\quad$ Machida, Nakamura, \& Matsumoto 2006; Johansen \& Levin 2008). Following the findings of these simulations, we separate the magnetic fields into mean fields, denoted by $\mathbf{B}=\left(0,<B_{\phi}>, 0\right)$, and the fluctuating fields, indicated as $\delta \mathbf{B}=\left(\delta B_{r}, \delta B_{\phi}, \delta B_{z}\right)$. Here, we express the azimuthal average by ' $<>$ ' and upon azimuthal averaging, the fluctuating components of the magnetic fields eventually disappear $(<\delta \mathbf{B}>=0)$. Moreover, the radial and vertical components of the magnetic field are assumed to be negligible when compared with the azimuthal component, $\left|\left\langle B_{\phi}\right\rangle+\delta B_{\phi}\right| \gg\left|\delta B_{r}\right|$ and $\left|\delta B_{z}\right|$. This ultimately renders the azimuthally averaged magnetic fields which is given by $\langle\mathbf{B}\rangle=\hat{\phi}\left\langle B_{\phi}\right\rangle$ (Oda et al. 2007).

\subsection{Model Equations}

In this work, a thin, axisymmetric, magnetized accretion flow onto a rotating black hole is considered and the accretion disc is assumed to lie on the black hole equatorial plane. Moreover, we employ the cylindrical polar coordinate $(x, \phi, z)$ to study the properties of accretion flow, where black hole is placed at its origin. In order to express the 
flow variables, we choose an unit system as $M_{\mathrm{BH}}=c=$ $G=1$, where $M_{\mathrm{BH}}$ is the mass of the black hole, $c$ represents the speed of light and $G$ denotes the gravitational constant, respectively. Accordingly, length, angular momentum and time are measured in units of $G M_{\mathrm{BH}} / c^{2}, G M_{\mathrm{BH}} / c$ and $G M_{\mathrm{BH}} / c^{3}$, respectively. In the subsequent sections, we choose $M_{\mathrm{BH}}=10 M_{\odot}$ as a reference value.

Considering steady state scenario, the governing equations of motion that describe the magnetized accreting matter are obtained as follows:

(i) Equation for radial momentum:

$$
v \frac{d v}{d x}+\frac{1}{\rho} \frac{d P}{d x}+\frac{d \Psi_{\mathrm{eff}}}{d x}+\frac{\left\langle B_{\phi}^{2}\right\rangle}{4 \pi x \rho}=0,
$$

where $v$ and $\rho$ stand for the radial velocity and density of the flow and $P$ represents total pressure which we take into account as $P=p_{\text {gas }}+p_{\text {mag }}$ where, $p_{\text {gas }}$ and $p_{\text {mag }}$ denote the gas pressure and the magnetic pressure of the flow. We obtain the gas pressure inside the disc as $p_{\text {gas }}=R \rho T / \mu$, where $R, T$ and $\mu$, respectively, represent the gas constant, the temperature and the mean molecular weight. Here, we use $\mu=0.5$ for fully ionized hydrogen. Further, the magnetic pressure is obtained as $p_{\text {mag }}=<B_{\phi}^{2}>/ 8 \pi$. We define $\beta=p_{\text {gas }} / p_{\text {mag }}$ and using this, we attain total pressure as $P=p_{\text {gas }}(1+\beta) / \beta$. Moreover, in equation (1), $\Psi_{\text {eff }}$ denotes the effective pseudo potential around a rotating black hole (Chakrabarti \& Mondal 2006) and is given by,

$$
\Psi_{\mathrm{eff}}=-\frac{\mathcal{Q}+\sqrt{\mathcal{Q}^{2}-4 \mathcal{P \mathcal { R }}}}{2 \mathcal{P}}
$$

where

$$
\begin{gathered}
\mathcal{P}=\frac{\epsilon^{2} \lambda^{2}}{2 x^{2}}, \\
\mathcal{Q}=-1+\frac{\epsilon^{2} \omega \lambda r^{2}}{x^{2}}+\frac{2 a_{k} \lambda}{r^{2} x}, \\
\mathcal{R}=1-\frac{1}{r-x_{0}}+\frac{2 a_{k} \omega}{x}+\frac{\epsilon^{2} \omega^{2} r^{4}}{2 x^{2}} .
\end{gathered}
$$

Here, $x$ represents the cylindrical radial distance and $r$ specifies spherical radial distance, respectively. Also, $\lambda$ stands for the specific angular momentum of the flow. In addition, we write $x_{0}=0.04+0.97 a_{k}+0.085 a_{k}^{2}, \omega=2 a_{k} /\left(x^{3}+a_{k}^{2} x+2 a_{k}^{2}\right)$ and $\epsilon^{2}=\left(x^{2}-2 x+a_{k}^{2}\right) /\left(x^{2}+a_{k}^{2}+2 a_{k}^{2} / x\right)$, where $\epsilon$ refers the redshift factor and $a_{k}$ denotes the spin of the black hole. It is to be noted that the adopted pseudo potential satisfactorily mimics the space-time geometry around rotating black hole for $a_{k} \lesssim 0.8$ (Chakrabarti \& Mondal 2006).

(ii) Mass flux conservation equation:

$$
\dot{M}=2 \pi x v \Sigma,
$$

where $\dot{M}$ specifies the accretion rate which we treat as global constant all throught and $\Sigma$ represents the vertically integrated density (Matsumoto et al. 1984). It may be noted that in this work, the direction of the inward radial velocity is considered as positive always.

(iii) Azimuthal momentum conservation equation:

$$
v \frac{d \lambda(x)}{d x}+\frac{1}{\Sigma x} \frac{d}{d x}\left(x^{2} T_{x \phi}\right)=0 .
$$

Here, we assume the vertically integrated total stress to be dominated by the $x \phi$ component of the Maxwell stress $T_{x \phi}$. For the accretion flow with large radial velocity, $T_{x \phi}$ comes out to be (Chakrabarti \& Das 2004; Machida, Nakamura, \& Matsumoto 2006)

$$
T_{x \phi}=\frac{<B_{x} B_{\phi}>}{4 \pi} h=-\alpha_{B}\left(W+\Sigma v^{2}\right),
$$

where $h, \alpha_{B}$ and $W$, respectively, represent the local disc height, the proportionality constant and the vertically integrated pressure of the flow (Matsumoto et al. 1984). Following the work of Shakura \& Sunvaev (1973), we regard $\alpha_{B}$ as a global constant all throughout of the flow. Note that when $v$ is significantly small, as in the case of Keplerian disc, equation (4) reduces to ' $\alpha$-model' (Shakura \& Sunvaev 1973).

We consider thin disc approximation where infalling matter maintains hydrostatic equilibrium in the vertical direction and calculate the disc height $(h)$ as, $h=$ $a \sqrt{x /\left(\gamma \Psi_{r}^{\prime}\right)}$ where $\Psi_{r}^{\prime}=\left(\frac{\partial \Psi_{\text {eff }}}{\partial r}\right)_{z<<x}, z$ denotes local vertical scale height in the cylindrical coordinate system and $r=\sqrt{x^{2}+z^{2}}$ (Das, Chakrabarti, \& Mondal 2010). Here, we define the sound speed as $a=\sqrt{\gamma P / \rho}$, where $\gamma$ stands for the adiabatic index of the flow. In this work, we assume $\gamma$ to remain constant along the flow and choose $\gamma=4 / 3$.

(iv) The equation for entropy:

$$
\Sigma v T \frac{d s}{d x}=\frac{h v}{\gamma-1}\left(\frac{d p_{\text {gas }}}{d x}-\frac{\gamma p_{\text {gas }}}{\rho} \frac{d \rho}{d x}\right)=Q^{-}-Q^{+},
$$

where $T$ and $s$ refer to the temperature and specific entropy of the flow, respectively. Moreover, $Q^{+}$ denotes the heating rate and $Q^{-}$represents the cooling rate of the flow. Meanwhile, the numerical simulation works of Hirose, Krolik, \& Stone (2006); Machida, Nakamura, \& Matsumoto (2006) indicate that during accretion, heating of the accreting matter occurs because of the energy dissipation via magnetic reconnection process and is calculated as

$$
Q^{+}=\frac{<B_{x} B_{\phi}>}{4 \pi} x h \frac{d \Omega}{d x}=-\alpha_{B}\left(W+\Sigma v^{2}\right) x \frac{d \Omega}{d x},
$$

where $\Omega$ stands for the angular velocity of the flow.

Usually, the accretion flow experiences heat loss as the consequences of the variety of cooling mechanisms, such as bremsstrahlung, synchrotron and Comptonization of bremsstrahlung as well as synchrotron photons. However, in the present study, as the infalling matter is magnetized in nature, we therefore consider only the synchrotron radiative mechanism as dominant cooling process and the corresponding cooling rate is obtained as (Shapiro \& Teukolsky 1983),

$$
Q^{-}=\frac{S a^{5} \rho h}{v} \sqrt{\frac{\Psi_{r}^{\prime}}{x^{3}}} \frac{\beta^{2}}{(1+\beta)^{3}},
$$

with,

$$
S=1.4827 \times 10^{18} \frac{\dot{m} \mu^{2} e^{4}}{I_{n} m_{e}^{3} \gamma^{5 / 2}} \frac{1}{G M_{\odot} c^{3}},
$$

where $e$ and $m_{e}$ represent the charge and mass of the electron and $\dot{m}$ denotes the accretion rate expressed in units of Eddington rate $\left(\dot{M}_{\mathrm{Edd}}=1.39 \times 10^{17} \times M_{\mathrm{BH}} / M_{\odot}\right.$ $\left.\mathrm{gm} \mathrm{s}^{-1}\right)$. Also, $I_{n}=\left(2^{n} n !\right)^{2} /(2 n+1)$ ! and $n$ represents the polytropic index of the flow which is related to the adiabatic index as $n=1 /(\gamma-1)$. We estimate the electron 
temperature employing the relation $T_{e}=\left(\sqrt{m_{e} / m_{p}}\right) T_{p}$, where the coupling between ion and electron is neglected (Chattopadhyay \& Chakrabarti 2002). Here, $m_{p}$ and $T_{p}$ refer the mass and temperature of the ion. Note that in this work, we ignore the bremsstrahlung emission process as it is an inefficient cooling process for stellar mass black hole system (Chattopadhvav \& Chakrabarti 2002). Moreover, we also disregard the inverse Comptonization process as well although its contribution may not be negligible especially at the inner part of the disc. Nevertheless, we make this assumption simply because the framework of single temperature accretion flow does not allow one to study the Componization process as it requires the consideration of twotemperature flow. However, we infer that when both synchrotron and Compton processes are present, the accretion flow will experience more dissipation and therefore, the results we present in the subsequent sections are expected to modify quantitatively although the overall conclusions perhaps be remain qualitatively unaltered.

(v) The advection equation of toroidal magnetic flux:

Following induction equation, the advection rate of toroidal magnetic flux is obtained as,

$$
\frac{\partial<B_{\phi}>\hat{\phi}}{\partial t}=\nabla \times\left(\vec{v} \times<B_{\phi}>\hat{\phi}-\frac{4 \pi}{c} \eta \vec{j}\right),
$$

where $\vec{v}, \vec{j}$ and $\eta$, respectively, represent the velocity vector, the current density and the resistivity of the flow. It may be noted that equation (8) is azimuthally averaged. For an accretion disc, since the Reynold number is generally very large, we ignore the magnetic-diffusion terms because of large length scale. Furthermore, here we ignore dynamo term as well. Considering steady state, the obtained equation is further vertically integrated employing the assumption that the azimuthally averaged toroidal magnetic fields disappear at disc surface. Based on these considerations, the toroidal magnetic flux advection rate is calculated as,

$$
\dot{\Phi}=-\sqrt{4 \pi} v h B_{0}(x)
$$

where

$$
\begin{aligned}
B_{0}(x) & =\left\langle B_{\phi}\right\rangle(x ; z=0) \\
& =2^{5 / 4} \pi^{1 / 4}(R T / \mu)^{1 / 2} \Sigma^{1 / 2} h^{-1 / 2} \beta^{-1 / 2}
\end{aligned}
$$

denotes azimuthally averaged toroidal magnetic field resided at the equatorial plane of the accretion disc (Oda et al. 2007). Inside the accretion disc, if the magnetic flux is dissipated by the magnetic reconnection or escapes from the disc due to buoyancy, $\dot{\Phi}$ will not be conserved. Besides, when MRI driven dynamo augments the toroidal magnetic flux, $\dot{\Phi}$ may vary with radial coordinate. Keeping these findings in mind, we thus consider $\dot{\Phi} \propto x^{-\zeta}$ (Oda et al. 2007), where $\zeta$ stands for a parameter describing the magnetic flux advection rate. Therefore, we have the following parametric relation as

$$
\dot{\Phi}(x ; \zeta, \dot{M}) \equiv \dot{\Phi}_{\text {edge }}\left(\frac{x}{x_{\text {edge }}}\right)^{-\zeta},
$$

where $\dot{\Phi}_{\text {edge }}$ indicates the advection rate of the toroidal magnetic field at a large distance, usually the disc outer edge $\left(x_{\text {edge }}\right)$. For $\zeta=0$, radial magnetic flux remains conserved whereas, for $\zeta>0$, the magnetic flux is increased with the decrease of $x$. However, for representation, in this study, we choose $\zeta=1$ all throughout unless stated otherwise.

\subsection{Analysis of transonic conditions}

During the course of accretion, matter from the outer edge of the disc $\left(x_{\text {edge }}\right)$ proceeds towards the black hole under the influence of gravity. In reality, inflowing matter possesses negligible radial velocity at $x_{\text {edge }}$ in contrast with the local sound speed and enters into the black hole with velocity equivalent to $c$. This findings evidently demand the transonic nature of the accreting matter. The radial coordinate where the accretion flow smoothly changes its sonic character from subsonic to supersonic state is commonly called as critical point. In order to analyze the transonic conditions, we simultaneously solve equations (1), (2), (3), (5), (9) and (10) and obtain the wind equation (Das 2007, and references therein) which is given by,

$$
\frac{d v}{d x}=\frac{N}{D}
$$

where the numerator $(N)$ is calculated as,

$$
\begin{gathered}
N=\frac{S a^{5}}{v} \sqrt{\frac{\Psi_{r}^{\prime}}{x^{3}}} \frac{\beta^{2}}{(1+\beta)^{3}}+\frac{2 \alpha_{B}^{2} I_{n}\left(a^{2} g+\gamma v^{2}\right)^{2}}{\gamma^{2} x v} \\
+\left[\frac{[3+\beta(\gamma+1)] v}{(\gamma-1)(1+\beta)}-\frac{4 \alpha_{B}^{2} g I_{n}\left(a^{2} g+\gamma v^{2}\right)}{\gamma v}\right]\left(\frac{d \Psi_{\text {eff }}}{d x}\right) \\
+\left[\frac{v a^{2}(2 \beta \gamma+4)}{2 \gamma(\gamma-1)(1+\beta)}-\frac{2 \alpha_{B}^{2} I_{n} a^{2} g\left(a^{2} g+\gamma v^{2}\right)}{\gamma^{2} v}\right]\left(\frac{d \ln \Psi_{r}^{\prime}}{d x}\right) \\
+\frac{2\{3+\beta(\gamma+1)\} a^{2} v}{\gamma x(\gamma-1)(1+\beta)^{2}}-\frac{3 a^{2} v(2 \gamma \beta+3)}{2 \gamma x(1+\beta)(\gamma-1)} \\
+\frac{6 \alpha_{B}^{2} I_{n} a^{2} g\left(a^{2} g+\gamma v^{2}\right)}{\gamma^{2} v x}-\frac{8 \alpha_{B}^{2} I_{n} a^{2} g\left(a^{2} g+\gamma v^{2}\right)}{\gamma^{2} v(1+\beta) x} \\
-\frac{a^{2} v(4 \zeta-1)}{2 \gamma(1+\beta)(\gamma-1) x}-\frac{4 \lambda \alpha_{B} I_{n}\left(a^{2} g+\gamma v^{2}\right)}{\gamma x^{2}}
\end{gathered}
$$

and the denominator $(D)$ is calculated as,

$$
\begin{gathered}
D=\frac{2 a^{2}(2+\gamma \beta)}{\gamma(\gamma-1)(1+\beta)}-\frac{\{3+\beta(\gamma+1)\} v^{2}}{(1+\beta)(\gamma-1)} \\
+\frac{2 \alpha_{B}^{2} I_{n}\left(a^{2} g+\gamma v^{2}\right)}{\gamma}\left[(2 g-1)-\frac{a^{2} g}{\gamma v^{2}}\right] .
\end{gathered}
$$

In the above analysis, we define $g=I_{n+1} / I_{n}$.

Next, we calculate the derivative of $a, \lambda$ and $\beta$ with respect to $x$ as,

$$
\begin{aligned}
\frac{d a}{d x}=- & \left(\frac{\gamma v}{a}-\frac{a}{v}\right) \frac{d v}{d x}+\frac{3 a}{2 x}-\frac{a}{2}\left(\frac{d \ln \Psi_{r}^{\prime}}{d x}\right) \\
& -\frac{\gamma}{a}\left(\frac{d \Psi_{\text {eff }}}{d x}\right)-\frac{2 a}{(1+\beta) x} \\
\frac{d \lambda}{d x}=- & \frac{\alpha_{B} x\left(a^{2} g-\gamma v^{2}\right)}{\gamma v^{2}} \frac{d v}{d x}+\frac{2 \alpha_{B} a x g}{\gamma v} \frac{d a}{d x}
\end{aligned}
$$




$$
\begin{gathered}
+\frac{\alpha_{B}\left(a^{2} g+\gamma v^{2}\right)}{\gamma v} \\
\frac{d \beta}{d x}=\left[\frac{4(1+\beta)}{v}-\frac{3 \gamma v(1+\beta)}{a^{2}}\right] \frac{d v}{d x}+\frac{9(1+\beta)}{2 x} \\
-2(1+\beta)\left(\frac{d \ln \Psi_{r}^{\prime}}{d x}\right)-\frac{3 \gamma(1+\beta)}{a^{2}} \frac{d \Psi_{\mathrm{eff}}}{d x} \\
-\frac{6}{x}+\frac{(1+\beta)(4 \zeta-1)}{2 x}
\end{gathered}
$$

Since the accretion solutions must be smooth along the streamline, the radial velocity gradient $(d v / d x)$ will be inevitably real and finite at every radial coordinate. Nevertheless, equation (11b) is revealed the fact that between $x_{\text {edge }}$ and the black hole horizon, there is a possibility where the denominator $(D)$ may vanish at some point. In order for maintaining the flow to become smooth always, it is therefore necessary that the location where $D$ goes to zero, $N$ also must vanish there. The location where $N$ and $D$ simultaneously disappears has a special significance and such location is termed as critical point $\left(x_{c}\right)$. It is to be noted that accretion flow becomes transonic at $x_{c}$ and accordingly, we have two conditions at $x_{c}$ which are obtained by setting $N=0$ and $D=0$, respectively. Using $D=0$, we calculate the Mach number (defined as the ratio of radial velocity to the sound speed, $M=v / a)$ at $x_{c}$ as,

$$
M_{c}=\sqrt{\frac{-m_{2}-\sqrt{m_{2}^{2}-4 m_{1} m_{3}}}{2 m_{1}}}
$$

where

$$
\begin{gathered}
m_{1}=2 \alpha_{B}^{2} I_{n} \gamma^{2}(\gamma-1)(2 g-1)\left(1+\beta_{c}\right)-\gamma^{2}\left\{3+(\gamma+1) \beta_{c}\right\} \\
m_{2}=2 \gamma\left(2+\gamma \beta_{c}\right)+4 \alpha_{B}^{2} I_{n} \gamma g(g-1)(\gamma-1)\left(1+\beta_{c}\right), \\
m_{3}=-2 \alpha_{B}^{2} I_{n} g^{2}(\gamma-1)\left(1+\beta_{c}\right) .
\end{gathered}
$$

Setting $N=0$, we obtain a cubic equation of sound speed $\left(a_{c}\right)$ at $x_{c}$ as,

$$
\mathcal{A} a_{c}^{3}+\mathcal{B} a_{c}^{2}+\mathcal{C} a_{c}+\mathcal{D}=0
$$

where

$$
\begin{gathered}
\mathcal{A}=S \sqrt{\frac{\Psi_{r}^{\prime}}{x_{c}^{3}}} \frac{\beta_{c}^{2}}{\left(1+\beta_{c}\right)^{3}}, \\
\mathcal{B}=\frac{2 \alpha_{B}^{2} I_{n}\left(g+\gamma M_{c}^{2}\right)^{2}}{\gamma^{2} x_{c}}+\frac{M_{c}^{2}\left(2 \gamma \beta_{c}+4\right)}{2 \gamma(\gamma-1)\left(1+\beta_{c}\right)}\left(\frac{d \ln \Psi_{r}^{\prime}}{d x}\right) \\
-\frac{2 \alpha_{B}^{2} I_{n} g\left(g+\gamma M_{c}^{2}\right)}{\gamma^{2}}\left(\frac{d \ln \Psi_{r}^{\prime}}{d x}\right) \\
+\frac{2\left\{3+\beta_{c}(\gamma+1)\right\} M_{c}^{2}}{\gamma x_{c}(\gamma-1)\left(1+\beta_{c}\right)^{2}} \\
-\frac{3 M_{c}^{2}\left(2 \gamma \beta_{c}+3\right)}{2 \gamma(\gamma-1)\left(1+\beta_{c}\right) x_{c}}+\frac{6 \alpha_{B}^{2} I_{n} g\left(g+\gamma M_{c}^{2}\right)}{\gamma^{2} x_{c}}
\end{gathered}
$$

$$
\begin{gathered}
-\frac{8 \alpha_{B}^{2} I_{n} g\left(g+\gamma M_{c}^{2}\right)}{\gamma^{2}\left(1+\beta_{c}\right) x_{c}}-\frac{(4 \zeta-1) M_{c}^{2}}{2 \gamma(\gamma-1)\left(1+\beta_{c}\right) x_{c}}, \\
\mathcal{C}=-\frac{4 \lambda_{c} \alpha_{B} I_{n} M_{c}\left(g+\gamma M_{c}^{2}\right)}{\gamma x_{c}^{2}}, \\
\mathcal{D}=\left[\frac{\left[3+\beta_{c}(\gamma+1)\right] M_{c}^{2}}{\left(1+\beta_{c}\right)(\gamma-1)}-\frac{4 \alpha_{B}^{2} g I_{n}\left(g+\gamma M_{c}^{2}\right)}{\gamma}\right] \\
\times\left(\frac{d \Psi_{\text {eff }}}{d x}\right) .
\end{gathered}
$$

Here, the flow variables specified using subscript ' $c$ ' denote their values evaluated at $x_{c}$.

Now, using the accretion flow parameters, we solve equation (16) to obtain the sound speed $\left(a_{c}\right)$ at $x_{c}$ and subsequently calculate $v_{c}$ using equation (15). By employing the values of $v_{c}$ and $a_{c}$ in Eq. (11), we examine the characteristics of the critical points. At the critical point, we get $(d v / d x)=0 / 0$ and thus, we use l'Hospital rule for obtaining the value of $(d v / d x)$ at $x_{c}$ (hereafter, $\left.(d v / d x)_{c}\right)$. Usually, $(d v / d x)_{c}$ owns two values; one for accretion and the other for wind. When the values of $(d v / d x)_{c}$ are real and of opposite sign, the critical point is known as saddle type (Chakrabarti \& Das 2004) and this type of critical point is particularly important due to the fact that transonic solution can cross it smoothly. In the present study, since our motivation is to investigate the structure of the magnetized accretion flow, we therefore focus into the accretion solutions only in the subsequent analysis.

\section{RESULTS AND DISCUSSIONS}

\subsection{Transonic Global Solutions}

In this work, we intend to obtain the global magnetized transonic accretion solution that delineates a smooth connection between horizon and the disc edge. With this aim, we simultaneously solve the equations (11-14) for a specified set of flow parameters. While doing this, we treat $\dot{m}$, $\alpha_{B}$, and $\gamma$ as global parameters of the flow. Moreover, one requires $a_{k}$ value and the boundary values of $\lambda$ and $\beta$ at a given $x$ as local parameters to solve these equations. Note that we express angular momentum $(\lambda)$ in terms of Keplerian angular momentum $\lambda_{\mathrm{K}}\left(\equiv \sqrt{x^{3} /(x-2)^{2}}\right)$ all throughout the paper. Since the black hole accretion solutions are necessarily transonic in nature, flow must pass through at least one critical point and therefore, it is reasonable to choose the boundary values of the flow at the critical point. With this, we hereby integrate equations (11-14) starting from the critical point once inwards up to just outside the black hole horizon and then outward up to a large distance (equivalently 'outer edge of the disc'). Ultimately, these two parts of are joined to obtain a complete global transonic accretion solution. Depending on the input parameters, accretion flow may possess single or multiple critical points Das, Chattopadhyav, \& Chakrabarti 2001a; Sarkar \& Das 2013). These critical points are classified as inner $\left(x_{\text {in }}\right)$ or outer $\left(x_{\text {out }}\right)$ critical points depending on whether they form close to or far away from the black hole horizon. 


\subsection{Global Accretion Solutions with Shock}

When the accretion flow containing multiple critical points accretes on to a black hole, it first passes through the outer critical point $\left(x_{\text {out }}\right)$ to become supersonic and keeps on accreting further inwards. Meanwhile, flow starts experiencing centrifugal repulsion resulting the accumulation of matter in the nearby region of the black hole that ultimately induces the shock transition when the density threshold is reached. With this, an effective virtual barrier is formed around the black hole. At shock, supersonic flow jumps in to the subsonic branch that makes the post-shock flow hot as the kinetic energy of the flow is converted to the thermal energy. Moreover, across the shock, flow undergoes shock compression that ultimately causes the post-shock flow to become dense. Interestingly, $2^{\text {nd }}$ law of thermodynamics suggests that shocked accretion solutions are favorable as the entropy of the post-shock matter is comparatively higher than the pre-shock matter (Becker \& Kazanas 2001). We calculate the entropy of the flow which is expressed as Chakrabarti 1996a $), \dot{\mathcal{M}}(x)=v x a^{2 n+1}\left(\frac{\beta}{1+\beta}\right)^{n} \sqrt{\frac{x}{\gamma \Psi_{r}^{\prime}}}$. In the dissipation free limit, $\dot{\mathcal{M}}$ remains constant all throughout expect at the shock transition. What is more is that at the discontinuous transition, the conservation of mass flux, momentum flux, energy flux and magnetic flux are held in order to satisfy the standing shock conditions (Sarkar \& Das 2016, and reference therein) and hence, these conservation laws across the shock front can be written as the continuity of (a) mass flux $\left(\dot{M}_{-}=\dot{M}_{+}\right)$(b) the momentum flux $\left(W_{-}+\Sigma_{-} v_{-}^{2}=W_{+}+\Sigma_{+} v_{+}^{2}\right)(\mathrm{c})$ the energy flux $\left(\mathcal{E}_{-}=\mathcal{E}_{+}\right)$ and $(\mathrm{d})$ the magnetic flux $\left(\dot{\Phi}_{-}=\dot{\Phi}_{+}\right)$, respectively. In this work, we consider the shock to be thin and non-dissipative and the flow variables with subscripts ' - ' and ' + ' represent their values just before and after the shock. Following Fukue (1990); Samadi, Abbassi, \& Khajavi (2014), we calculate the local energy of the magnetized dissipative accretion flow as $\mathcal{E}(x)=v^{2} / 2+a^{2} /(\gamma-1)+\Psi_{\text {eff }}+<B_{\phi}^{2}>/(4 \pi \rho)$, where all the above quantities bear their usual meaning. In the subsequent analysis, upon employing the above set of shock conditions, we compute the shock position and its diverse properties knowing the input parameters of the accretion flow.

In Fig. 1, we show the result obtained from one representative case where the variation of Mach number $(M=$ $v / a$ ) with the logarithmic radial distance is depicted. We choose the injection parameters of the flow at the outer edge $\left(x_{\text {edge }}=1000\right)$ as $\mathcal{E}_{\text {edge }}=1.0793 \times 10^{-3}, \beta_{\text {edge }}=1.6 \times 10^{5}$, $\alpha_{B}=0.02$ and $\dot{m}=0.05$, respectively. In Fig. 1(a), we consider the black hole to be slowly rotating having $a_{k}=0.32$ and the flow is injected with angular momentum, $\lambda_{\text {edge }}=$ $0.124 \lambda_{\mathrm{K}}$. Here, flow is subsonic at the outer edge and becomes supersonic after crossing the outer critical point located at $x_{\text {out }}=530.90$. The supersonic flow proceeds further inwards and encounters shock transition at $x_{s}=16.20$ while jumping in to the subsonic branch. In the figure, shock position is shown using vertical arrow. Gradually flow velocity is increased as it moves inward and then it passes $x_{\text {in }}$ smoothly at 4.1777 before crossing the horizon. Here, we show the direction of the flow motion using arrows and mark the inner and outer critical points with filled circles. Next, we intend to examine the role of black hole spin in deciding the shock

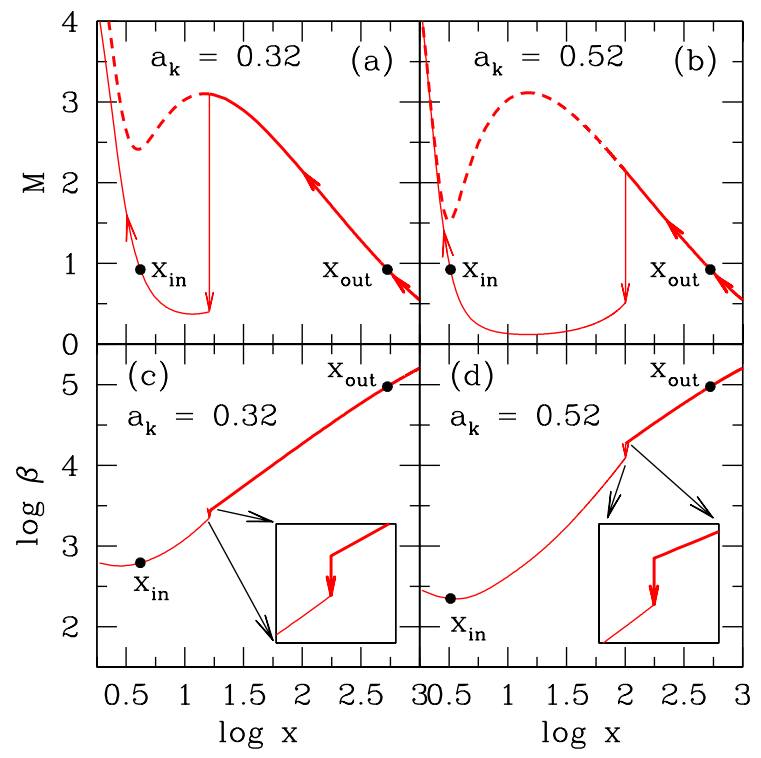

Figure 1. (a-b) Plot of Mach number with logarithmic radial distance. Flow is injected with $x_{\text {edge }}=1000, \lambda_{\text {edge }}=0.124 \lambda_{\mathrm{K}}$, $\mathcal{E}_{\text {edge }}=1.0793 \times 10^{-3}, \beta_{\text {edge }}=1.6 \times 10^{5}, \alpha_{B}=0.02$ and $\dot{m}=0.05$, respectively. We choose $a_{k}=0.32$ in panel (a) and $a_{k}=0.52$ in panel (b). (c-d) Logarithmic variation of plasma- $\beta$ corresponding to solutions (a) and (b). In each panel, $x_{\text {in }}$ and $x_{\text {out }}$ are indicated using filled circles and shock transition is shown by vertical arrow. See text for details.

transition and hence we inject matter on to a moderately rotating black hole $\left(a_{k}=0.52\right)$ keeping all the flow parameters same as in Fig. 1a. It may be noted that for this chosen set of flow parameters, standing shock solution ceases to exist when $a_{k}>0.52$. The result is depicted in Fig. 1b, where the outer critical point, shock location and inner critical point are obtained as $x_{\text {out }}=531.43, x_{\mathrm{s}}=100.62$ and $x_{\text {in }}=3.2502$, respectively. Since all the flow parameters at the outer edge of the disc are kept fixed including angular momentum, we observe that shock forms at larger radial distance for $a_{k}=0.52$. In reality, a spinning black hole distorts the space-time fabric in its vicinity, allowing matter to orbit at a closer distance as compared to a non-rotating one. Due to the effect of frame dragging, the fluid angular momentum is affected by the rotation of the black hole. It is known that the shock formation in accretion flow happens as a result of the competition between the gravitational pull and the centrifugal repulsion. When flow is injected from the outer edge of the disc with fixed boundary conditions, because of the spin-orbit coupling term in the Kerr geometry, the increase of spin parameter $\left(a_{k}\right)$ modifies the angular momentum profile of the flow and the shock front is pushed away from the horizon as is observed in Fig. 1. This finding is consistent with the results of Aktar. Das, \& Nandi 2015). Overall, we see that the standing shock in magnetized flow is continue to exist around the rotating black hole and when $a_{k}$ is increased, shock transition occurs for relatively low angular momentum flow and vice versa. Further, in panel (c) and (d), we show the variation of plasma- $\beta$ with $\log x$ corresponding to solutions presented in panel (a) and (b), respectively. In both the cases, we find that plasma- $\beta$ steadily 

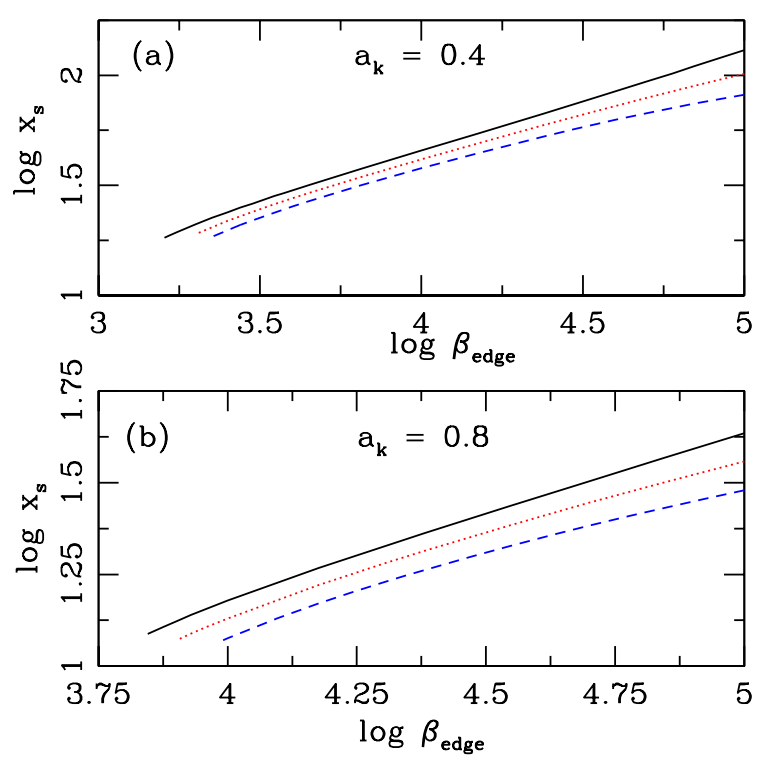

Figure 2. Shock location $\left(x_{s}\right)$ variation with $\beta_{\text {edge }}$. Here, the inflow parameters are chosen as $x_{\text {edge }}=1000, \mathcal{E}_{\text {edge }}=1.0793 \times$ $10^{-3}, \alpha_{B}=0.02$ and $\dot{m}=0.05$, respectively. In every panel, spin of the black hole $\left(a_{k}\right)$ is marked. In panel (a), results plotted with solid, dotted and dashed curves are obtained for $\lambda_{\text {edge }}=$ $0.12845 \lambda_{\mathrm{K}}, 0.12791 \lambda_{\mathrm{K}}$ and $0.12737 \lambda_{\mathrm{K}}$. And in panel (b), results depicted with solid, dotted and dashed curves are for $\lambda_{\text {edge }}=$ $0.11443 \lambda_{\mathrm{K}}, 0.11390 \lambda_{\mathrm{K}}$ and $0.11336 \lambda_{\mathrm{K}}$, respectively. See text for details.

decreases with the decrease of radial coordinate. This clearly indicates that the magnetic activity inside the disc increases as the flow accretes towards the horizon.

\subsection{Properties of Standing Shocks}

One of the pertinent aspect in understanding the magnetically supported accreting flow around the rotating black holes is to study the dependence of the shock position $\left(x_{s}\right)$ on the $\beta$ values. Accordingly, we calculate $x_{s}$ in terms of $\beta_{\text {edge }}$ for flows with fixed outer boundary values accreting on to a given black hole. For that, we choose the outer boundary parameters as $x_{\text {edge }}=1000, \mathcal{E}_{\text {edge }}=1.0793 \times 10^{-3}$, $\alpha_{B}=0.02$ and $\dot{m}=0.05$. In Fig. 2(a), we display the results obtained for $a_{k}=0.4$, where solid, dotted and dashed curves are for $\lambda_{\text {edge }}=0.12845 \lambda_{\mathrm{K}}, 0.12791 \lambda_{\mathrm{K}}$ and $0.12737 \lambda_{\mathrm{K}}$, respectively. We notice that the shock front proceeds towards the horizon with the decrease of $\beta_{\text {edge }}$ irrespective to the values of $\lambda_{\text {edge }}$. This happens because when $\beta_{\text {edge }}$ is decreased, the efficiency of synchrotron cooling is enhanced due to the increase of magnetic activity inside the disc. The effect becomes more prominent at the inner part of the disc (i.e., PSC) as, due to shock transition, both density and temperature are relatively higher there compared to the pre-shock flow. This renders the thermal pressure to drop down in the PSC region and ultimately shock front moves inward to maintain the pressure balance across it. Incidentally, keeping the all the boundary flow parameters fixed, one can not reduce $\beta_{\text {edge }}$ indefinitely as shock ceases to exist when $\beta_{\text {edge }}<\beta_{\text {edge }}^{\text {cri }}($ shock conditions fail to satisfy there).
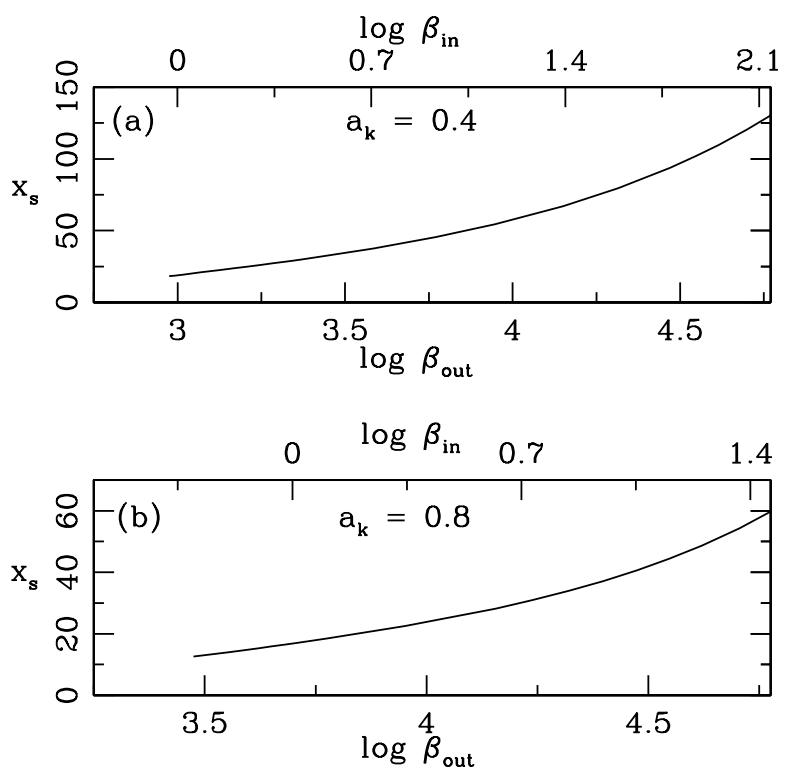

Figure 3. Variation of the shock location $\left(x_{s}\right)$ as function of $\beta_{\text {out }}$ (lower axis) and $\beta_{\text {in }}$ (upper axis). In each panel, $a_{k}$ is marked. See text for details.

It may be noted that $\beta_{\text {edge }}^{\text {cri }}$ does not have a universal value, instead it depends on the flow parameters fixed at the outer edge of the disc. Further, we depict the results for $a_{k}=0.8$ in Fig. 2b, where solid, dotted and dashed curves represent results corresponding to $\lambda_{\text {edge }}=0.11443 \lambda_{\mathrm{K}}, 0.11390 \lambda_{\mathrm{K}}$ and $0.11336 \lambda_{\mathrm{K}}$, respectively. Here, we keep all the other flow parameters same as in Fig. 2a. We find that the shock location proceeds towards the horizon with the decrease of $\beta_{\text {edge }}$ in all cases as observed in Fig. 2a.

Next, we examine the correlation of $\beta$ values between the inner and outer critical points for shock induced global accretion solutions. While doing this, we choose two cases where inflowing matters are accreted on to rotating black holes having different spin parameters as $a_{k}=0.4$ and 0.8 , respectively. For $a_{k}=0.4$, we consider the result depicted in Fig. 3a corresponding to $\lambda_{\text {edge }}=0.12845 \lambda_{\mathrm{K}}$ and show the variation of shock location as function of both $\beta_{\text {out }}$ (lower horizontal axis) and $\beta_{\text {in }}$ (upper horizontal axis). The other flow parameters are considered same as in Fig. 2. Here, $\beta_{\text {in }}$ and $\beta_{\text {out }}$ refer $\beta$ values measured at $x_{\text {in }}$ and $x_{\text {out }}$, respectively. We see that $x_{s}$ decreases when the magnetic activity is increased ( $\beta$ is decreased) inside the disc. We continue our study choosing the result presented in Fig. $3 \mathrm{~b}$ for $\lambda_{\text {edge }}=0.11551 \lambda_{\mathrm{K}}$ and show the variation of $x_{s}$ in terms of $\beta_{\text {out }}$ as well as $\beta_{\text {in }}$ in Fig. 3b. We observe that in all cases, $\beta_{\text {in }}<\beta_{\text {out }}$ all throughout. This finding is not surprising because, in our model, the advection of magnetic flux increases as the inflowing matter approaches towards the horizon and eventually, $\beta$ is reduced towards the inner part of the disc. Moreover, we find that shock solutions exist even for $\beta_{\text {in }}<1$ irrespective to the choice of $a_{k}$ value. This evidently indicates that global transonic accretion solutions harbor standing shock waves both in gas pressure dominated as well as in magnetic pressure dominated flows for a wide range of $a_{k}$ values.

It is worthy to explore the effect of cooling on the 

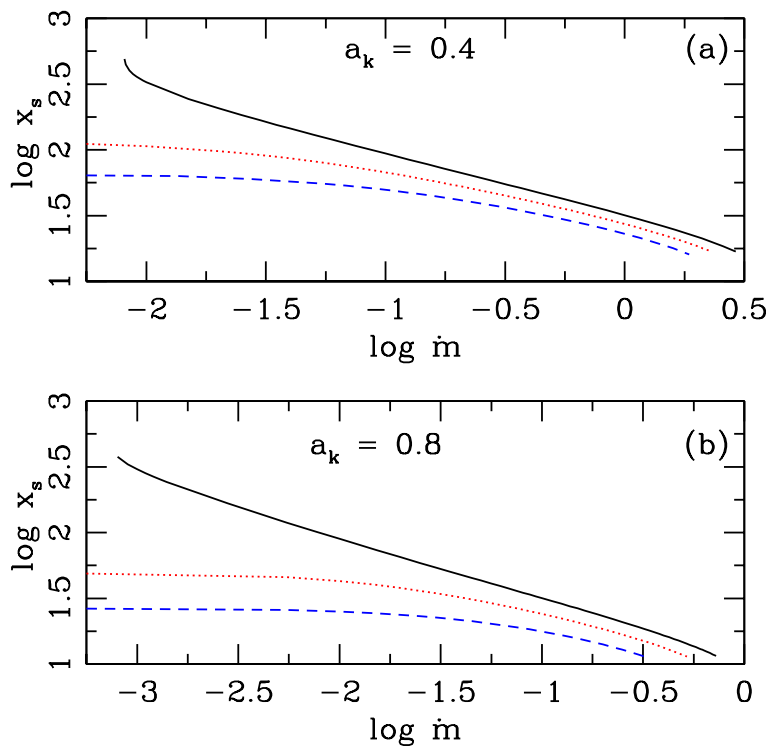

Figure 4. Variation of the shock location $\left(x_{s}\right)$ as function of $\dot{m}$. Flow parameters at the outer edge of the disc is chosen as $x_{\text {edge }}=1000, \mathcal{E}_{\text {edge }}=1.0793 \times 10^{-3}, \alpha_{B}=0.02$ and $\beta_{\text {edge }}=$ $10^{5}$, respectively. Results depicted in top and bottom panels are for $a_{k}=0.4$ and 0.8. In (a), solid, dotted and dashed curves are obtained for $\lambda_{\text {edge }}=0.12845 \lambda_{\mathrm{K}}, 0.12737 \lambda_{\mathrm{K}}$ and $0.12630 \lambda_{\mathrm{K}}$ whereas in (b), solid, dotted and dashed curves represents results for $\lambda_{\text {edge }}=0.11443 \lambda_{\mathrm{K}}, 0.11336 \lambda_{\mathrm{K}}$ and $0.11229 \lambda_{\mathrm{K}}$. See text for details.

formation of shock wave in an accretion flow and therefore, in Fig. 4, we study the variation of shock location $\left(x_{s}\right)$ with accretion rate $(\dot{m})$. Towards this, we consider the flow injection parameters as $x_{\text {edge }}=1000, \beta_{\text {edge }}=10^{5}$, $\mathcal{E}_{\text {edge }}=1.0793 \times 10^{-3}$ and $\alpha_{B}=0.02$, respectively. As before, in Fig. 4a, we chose $a_{k}=0.4$ and the profile of shock location $\left(x_{s}\right)$ is presented for various values of $\lambda_{\text {edge }}$. Here, solid, dotted and dashed curves represent flows injected with $\lambda_{\text {edge }}=0.12845 \lambda_{\mathrm{K}}, 0.12737 \lambda_{\mathrm{K}}$ and $0.12630 \lambda_{\mathrm{K}}$, respectively. From the figure, it is clear that large range of $\dot{m}$ admits standing shock in magnetized accretion flow. Moreover, we find that for a given $\lambda_{\text {edge }}, x_{s}$ moves inwards as $\dot{m}$ is increased. In reality, enhanced accretion rate boosts the efficiency of the radiative cooling that causes the flow to lose energy during accretion. Since PSC is hot and dense, the effect of cooling at PSC becomes profound that evidently decreases the post-shock thermal pressure. Consequently, this compels the shock front to settle down at some smaller distance to fulfill the shock conditions. Unfortunately, $\dot{m}$ can not be increased indefinitely due to the fact that when $\dot{m}$ exceeds its critical value $\left(\dot{m}^{\text {cri }}\right)$, standing shocks are no longer feasible as the shock conditions fail to satisfy there. Clearly, $\dot{m}^{\text {cri }}$ does not retain a global value, rather it depends on the flow parameters. It is also apparent that the possibility of standing shock formation reduces with the increase of $\dot{m}$. Furthermore, it is intriguing to understand what happens to the flow when standing shock conditions fail to satisfy. Interestingly, in that case, inner part of the accretion flow may start to modulate exhibiting the feature of oscillatory shock (Das \& Aktar 2015, and references therein). Unfor-
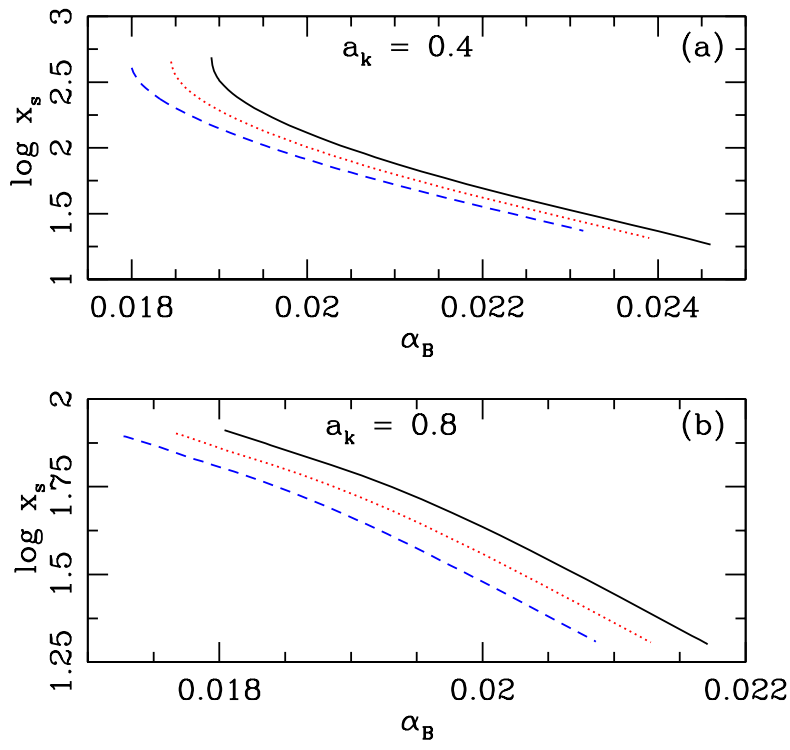

Figure 5. Variation of the shock location $\left(x_{s}\right)$ as function of $\alpha_{B}$. Accreting matter is supplied with inflow parameters as $x_{\text {edge }}=1000$, with $\mathcal{E}_{\text {edge }}=1.0793 \times 10^{-3}, \dot{m}=0.05$ and $\beta_{\text {edge }}=$ $10^{5}$, respectively. In each panel, $a_{k}$ is marked. In top panel (a), the results corresponding to $\lambda_{\text {edge }}=0.12845 \lambda_{\mathrm{K}}, 0.12791 \lambda_{\mathrm{K}}$ and $0.12737 \lambda_{\mathrm{K}}$ are represented using solid, dotted and dashed line style. The same line style is used to denote the results for $\lambda_{\text {edge }}=0.11443 \lambda_{\mathrm{K}}, 0.11390 \lambda_{\mathrm{K}}$ and $0.11336 \lambda_{\mathrm{K}}$ in lower panel (b). See text for details.

tunately, the investigation of non-steady shock properties is beyond the scope of the present paper. In addition, we find that for a given $\dot{m}$, shock front recedes away from the black hole when $\lambda_{\text {edge }}$ is increased. In reality, the discontinuous shock transition is essentially the manifestation of the competition between centrifugal repulsion and gravity. When $\lambda_{\text {edge }}$ is higher, accretion flow possesses higher angular momentum that causes the enhanced centrifugal repulsion against gravity. Because of this, shock front is pushed further out when $\lambda_{\text {edge }}$ is increased. This findings establishes the fact that shocks are centrifugally driven. In Fig. 4(b), we present the result corresponding to $a_{k}=0.8$, where solid, dotted and dashed curves represent results obtained for $\lambda_{\text {edge }}=0.11443 \lambda_{\mathrm{K}}, 0.11336 \lambda_{\mathrm{K}}$ and $0.11229 \lambda_{\mathrm{K}}$, respectively. Here also, we observe that the formation of shock and its dependence on $\dot{m}$ and $\lambda_{\text {edge }}$ are in general similar to the results shown in Fig. 4(a).

For completeness, we investigate the variation of shock location in terms of viscosity $\left(\alpha_{B}\right)$ for flows having fixed outer edge boundary parameters. Here, we choose the flow injection parameters as $x_{\text {edge }}=1000, \mathcal{E}_{\text {edge }}=1.0793 \times 10^{-3}$, $\beta_{\text {edge }}=10^{5}$ and $\dot{m}=0.05$, respectively. In Fig. 5 a, we show the obtained results for $a_{k}=0.4$, where solid, dotted and dashed curves are for $\lambda_{\text {edge }}=0.12845 \lambda_{\mathrm{K}}, 0.12791 \lambda_{\mathrm{K}}$ and $0.12737 \lambda_{\mathrm{K}}$, respectively. Notice that shocked accretion solutions exist for a wide range of $\alpha_{B}$ and shock location shifts towards the horizon with the increase of $\alpha_{B}$ for all cases having different $\lambda_{\text {edge }}$ values. In reality, as $\alpha_{B}$ is increased, angular momentum transport in the outward direction becomes more efficient that causes the weakening of centrifugal repul- 


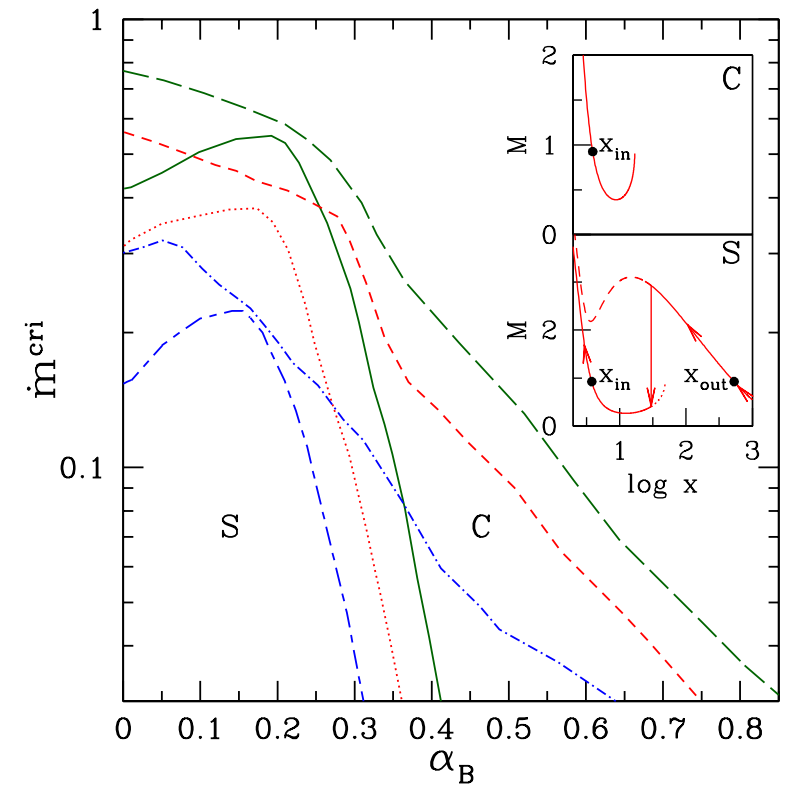

Figure 6. Variation of critical accretion rate $\left(\dot{m}^{\text {cri }}\right)$ as a function of viscosity parameter $\left(\alpha_{B}\right)$ for various $a_{k}$. Here, we choose $\beta_{\text {in }}=10$. Long-dashed, dashed and dot-dashed curves are obtained for $a_{k}=0,0.4$ and 0.8 and the region bounded by them in $\alpha_{B}-\dot{m}^{\text {cri }}$ plane provides closed accretion solutions passing through the inner sonic point. In addition, solid, dotted and shortlong-dashed curves represent the effective region corresponding to $a_{k}=0,0.4$ and 0.8 that admits standing shock solutions. In the inset, examples of closed (marked with C) and shocked solutions (marked with S) are presented. See text for details.

sion and hence, shock front is driven inward. When $\alpha_{B}$ exceeds its critical limit $\left(\alpha_{B}^{\mathrm{cri}}\right)$, shock conditions do not remain favorable and as a result, standing shock disappears. Again, it may be noted that $\alpha_{B}^{\text {cri }}$ largely depends on the accretion flow parameters. Further, in Fig. 5b, we display the result for $a_{k}=0.8$, where solid, dotted and dashed curves denote results for $\lambda_{\text {edge }}=0.11443 \lambda_{\mathrm{K}}, 0.11390 \lambda_{\mathrm{K}}$ and $0.11336 \lambda_{\mathrm{K}}$, respectively. Here, we find that shock location decreases with the increase of $\alpha_{B}$ around rotating black holes as well.

\subsection{Parameter Space for Shock}

We have already mentioned that during the course of accretion, inflowing matter may contain shock wave provided it possesses multiple critical points. Interestingly, one can obtain standing shock solution, if the standing shock conditions are satisfied (see §3.2). But, when shock conditions are not favorable and the entropy content at the inner critical point is higher than the outer critical point, the shock formation never remains steady as the shock location becomes imaginary (Das, Chattopadhyay, \& Chakrabarti 2001a) and therefore, shock starts to execute continuous back and forth movements that seems to exhibit the quasi-periodic oscillation phenomenon Das, Chattopadhyay, \& Chakrabarti 2001a). In this case, accretion solution passing through the inner critical point fails to connect the black hole horizon to the outer edge of the disc as it becomes closed in the range $x_{\text {in }}<x<x_{\text {out }}$ with $M(x)=M_{c}$ Chakrabarti \& Das 2004). Needless to mention that it is not possible to exam- ine the characteristics of the non-steady shock solution in the framework of the present paper, however, we estimate the critical accretion rate $\left(\dot{m}^{\text {cri }}\right)$ that provides accretion solutions containing standing shocks and/or closed topologies. While doing this, we fix $\beta_{\text {in }}=10$, and for a given $a_{k}$, we calculate $\dot{m}^{\text {cri }}$ as function of $\alpha_{B}$, where $x_{\text {in }}$ and $\lambda_{\text {in }}$ are varied freely. Accordingly, in Fig. 6, we classify the parameter space spanned by $\alpha_{B}$ and $\dot{m}^{\text {cri }}$ that provides closed topologies and standing shocks, respectively. Examples of closed topology (marked as C) and standing shock solution (marked as S) are displayed in the small boxes, where the variation of Mach number with radial coordinate is plotted. In the figure, longdashed, short-dashed and dot-dashed curves are obtained for $a_{k}=0,0.4$ and 0.8 that separate the $\alpha_{B}-\dot{m}^{\text {cri }}$ plane where left-bottom region allows closed topologies. Similarly, solid, dotted and short-long-dashed curves separate the standing shock parameter space for $a_{k}=0,0.4$ and 0.8 , respectively. We observe that the shock parameter space appears to be the subset of parameter space for closed topology all throughout. This is expected as the region of closed topologies includes the region of standing as well as oscillating shocks. Meanwhile, Das \& Chakrabarti (2008) showed that for fixed $a_{k}$, the effective region of standing shock parameter space shrinks with the increase of accretion rate for an inviscid flow. Actually, when the accretion rate is enhanced, cooling becomes more effective and hence, inflowing matter loses energy during accretion. On the other hand, viscosity enhances the flow energy as it accretes due to viscous heating. Interestingly, when both dissipation processes, namely, viscosity and synchrotron cooling, are present in the flow, viscous dissipation effectively compensates a part of the energy loss happens due to cooling. Here, in a way, viscosity and cooling act oppositely in deciding the shock parameter space. However, as synchrotron cooling and viscous heating depend differently on the flow variables, one does not cancel the other effect completely (Das 2007). Overall, for a given $a_{k}$, standing shock continues to form until an optimum combination of $\left(\alpha_{B}, \dot{m}^{\text {cri }}\right)$ is reached which evidently exhibits as a peak in the $\alpha_{B}-\dot{m}^{\text {cri }}$ plane. In general, the flow is dominated by cooling in the left side of the peak whereas viscous heating dominates on the other side. As expected, shock disappears when viscosity exceeds its critical limit (Chakrabarti \& Das 2004). In addition, in case of a rapidly rotating black hole, shock forms in a relatively low angular momentum accretion flow (Aktar. Das, \& Nandi 2015) that effectively causes the weak centrifugal repulsion and therefore, standing shock settles down at a smaller length scale. Moreover, when the level of dissipation is increased (namely, the increase of $\alpha_{B}$ and $\dot{m}$ ), shock front is compelled to move towards the horizon (see Fig. 4-5). This clearly indicates that rapidly rotating black holes can sustain shocks for lower dissipation rates and we observe the similar findings in Fig. 6.

Now, we intend to study the effect of magnetic fields in deciding the effective region of parameter space in $\left(\alpha_{B}, \dot{m}^{\text {cri }}\right)$ plane for standing shock. In Fig. 7, we present the obtained results, where shock parameter space is computed for rapidly rotating black hole $\left(a_{k}=0.8\right)$ considering different $\beta_{\text {in }}$ values. In the figure, the regions bounded with solid, dotted, short-dashed and long-dashed curves are obtained for $\beta_{\text {in }}=5,10,50$ and 100, respectively. We observe that the effective region of the parameter space for shock gradually reduces with the decrease of $\beta_{\text {in }}$. This happens 


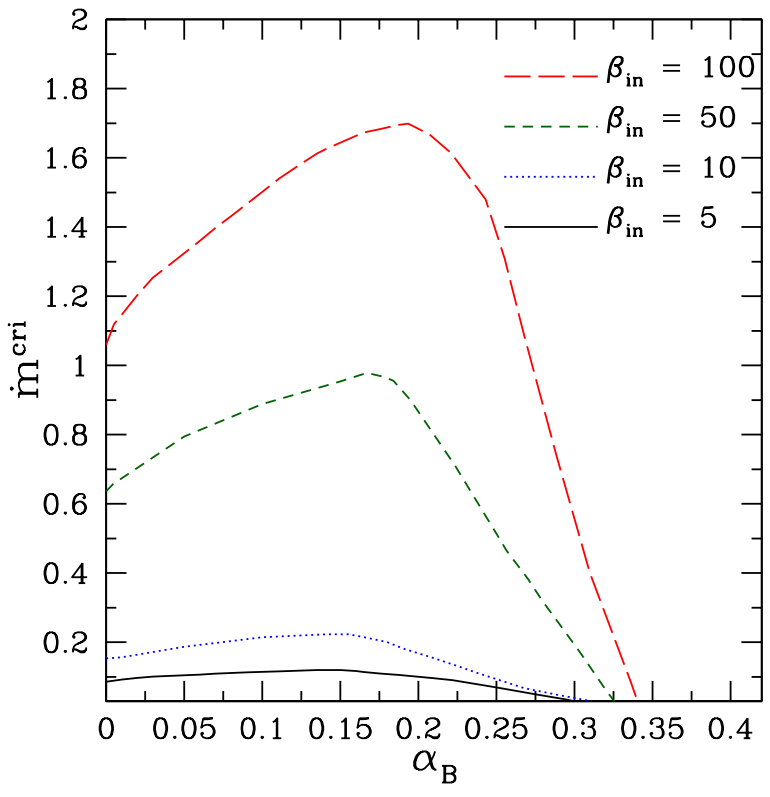

Figure 7. Variation of critical accretion rate $\left(\dot{m}^{\text {cri }}\right)$ for standing accretion shock with viscosity parameter $\left(\alpha_{B}\right)$ for different $\beta_{\text {in }}$. Here, we fix black hole spin as $a_{k}=0.8$. Solid, dotted, dashed and long-dashed curves denote results for $\beta_{\text {in }}=5,10,50$, and 100 , respectively. See text for details.

due to the fact that when $\beta_{\text {in }}$ is low, synchrotron cooling becomes very much effective and therefore, the level of dissipation experienced by the inflowing matter turns out to be significant even with moderate accretion rates. Thus, the possibility of shock formation is eventually reduced as the magnetic activity is increased inside the disc.

We carry out the analysis further to calculate the critical accretion rate $\left(\dot{m}^{\text {cri }}\right)$ of the flow as function of $\beta_{\text {in }}$ that provides global accretion solutions containing standing shock. In Fig. 8, we compare the critical accretion rate $\left(\dot{m}^{\text {cri }}\right)$ where solid and dashed curves represent the results obtained for non-rotating $\left(a_{k}=0\right)$ and rapidly rotating $\left(a_{k}=0.8\right)$ black holes, respectively. Here, we choose the viscosity parameter as $\alpha_{B}=0.01$. We find that standing shocks exist for a wide range of $\beta_{\text {in }}$ that effectively includes both gas pressure dominated flows $(\beta>1)$ as well as magnetic pressure dominated flows $(\beta<1)$. Since synchrotron process directly depends on the density and magnetic fields of the flow, one can achieve the desired cooling efficiency by suitably adjusting the accretion rate and plasma $\beta$. In the figure, we observe this findings for both the cases (for $a_{k}=0$ and 0.8 ) where the critical accretion rate $\left(\dot{m}^{\text {cri }}\right)$ for shock is found to be increased with $\beta_{\text {in. }}$. In reality, when $\beta_{\text {in }}<1$, the inner part of the disc is magnetically dominated and a tiny amount of accretion rate is sufficient to cool the flow. On the other hand, as $\beta_{\text {in }}$ is gradually increased, the strength of magnetic fields becomes weak and therefore, enhanced accretion rate is needed for the cooling of the flow. Interestingly, when $\beta_{\text {in }} \gg 1$, magnetic fields becomes insignificant and flow is capable of sustaining standing shocks even for super-Eddington accretion rates $\left(\dot{m}^{\text {cri }}>1\right)$. Moreover, we find that for a given $\beta_{\mathrm{in}}, \dot{m}^{\text {cri }}$ is smaller for higher $a_{k}$. This clearly indicates that inflowing matter around rapidly rotat-

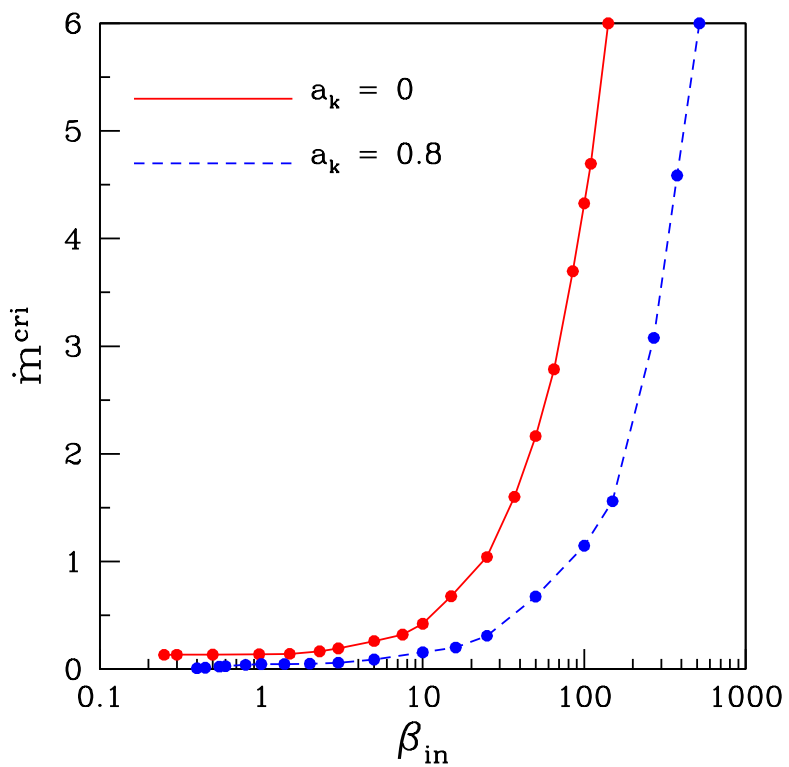

Figure 8. Comparison of critical accretion rate $\dot{m}^{\text {cri }}$ for shock with $\beta_{\text {in }}$. In the plot, filled circles joined with solid line denote results for $a_{k}=0$ and filled circles connected with dashed line represent results corresponding to $a_{k}=0.8$, respectively. Here $\alpha_{B}=0.01$ is used. See text for details.

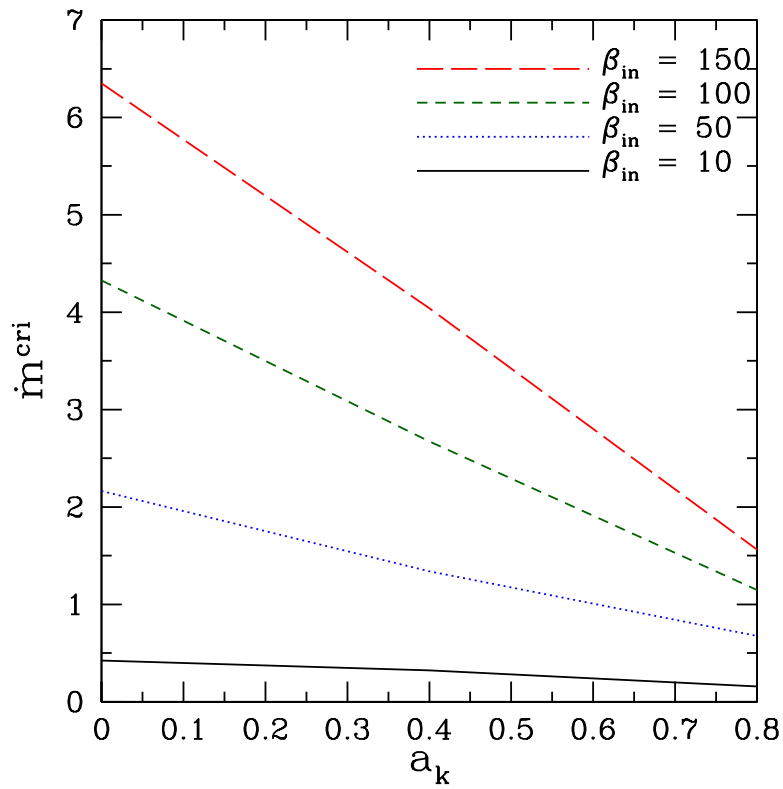

Figure 9. Variation of critical accretion rate $\dot{m}^{\text {cri }}$ with $a_{k}$ for shock. Here, we fix viscosity parameter as $\alpha_{B}=0.01$. Results depicted with solid, dotted, dashed and big-dashed line style correspond to $\beta_{\text {in }}=10,50,100$ and 150 . See text for details.

ing black holes contain shocks for relatively lower accretion rates which is consistent with the findings of Fig. 6 .

In the context of the formation of standing shock in an magnetized accretion flow, we now illustrate the dependence of the critical accretion rate $\left(\dot{m}^{\text {cri }}\right)$ on the spin of the black hole $\left(a_{k}\right)$ in Fig. 9. In order for that we fix the viscosity as $\alpha_{B}=0.01$. Here, solid, dotted, dashed and long-dashed 


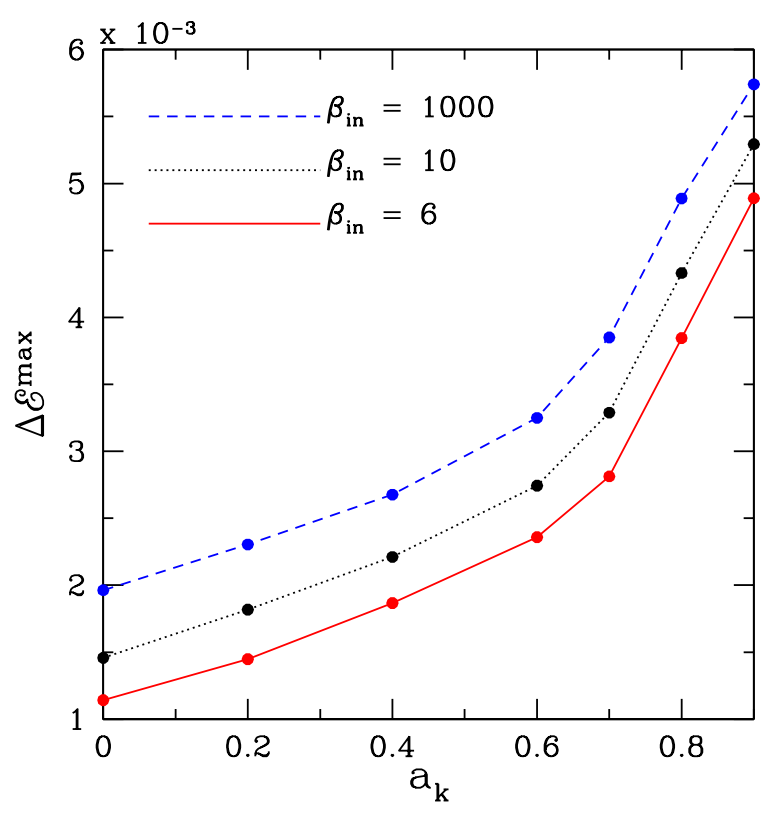

Figure 10. Plot of maximum energy dissipation $\left(\Delta \mathcal{E}^{\max }\right)$ at the shock with $a_{k}$ for three distinct values of $\beta_{\text {in }}$. Here, we choose accretion rate as $\dot{m}=0.05$ and fix viscosity parameter as $\alpha_{B}=$ 0.01. Solid, dotted and dashed curves are obtained for $\beta_{\text {in }}=6,10$ and 1000 , respectively. See text for details.

curves are obtained for $\beta_{\text {in }}=10,50,100$ and 150, respectively. We observe that for a given $\beta_{\text {in }}, \dot{m}^{\text {cri }}$ decreases with the increase of $a_{k}$ in all cases. Moreover, here again we find that when $\beta_{\text {in }}$ is large, accretion flow continues to sustain standing shock for higher accretion rate and vice versa.

\subsection{Energy Extraction from PSC}

So far, we have carried out the investigation of standing shock properties for flows accreting on to rotating black holes. While doing this, we consider the shock to be thin and non-dissipative and therefore, the specific energy remains essentially conserved across the shock front Chakrabarti 1989). However, in reality, the nature of the shock can be dissipative as well and in that case, the available energy dissipated at shock escaped through the disc surfaces along the vertical direction. A part of this energy is then converted to hard radiations and the rest may be used in jet generation as jets seem to be originated from the PSC around rotating black holes Aktar et al. 2017, and references therein). In effect, this cause the depletion of energy at PSC (Singh \& Chakrabarti 2011). Moreover, Chakrabarti \& Titarchuk (1995) pointed out that the dissipative energy at shock is likely to be regulated via thermal Comptonization process that ultimately reduces the thermal energy of the PSC. Based on the above insight, we model the dissipated energy to be proportional to the temperature difference between the immediate pre-shock and post-shock flow. Following this, the energy loss $(\Delta \mathcal{E})$ at the shock is estimated as (Das. Chakrabarti, \& Mondal 2010),

$$
\Delta \mathcal{E}=f n\left(a_{+}^{2}-a_{-}^{2}\right)
$$

where $a_{-}$and $a_{+}$specify the sound speed just before and after the shock transition. Here, $f$ refers the fractional value of thermal energy difference dissipated at shock and we treat it as free parameter (Das, Chakrabarti, \& Mondal 2010; Sarkar \& Das 2013; Kumar \& Chattopadhvav 2013; Sarkar, Das, \& Mandal 2018). For the purpose of representation, in this work, we choose $f=0.1$ all throughout.

In Fig. 10, we show how the maximum energy dissipated at shock $\left(\Delta \mathcal{E}^{\max }\right)$ is varied with $a_{k}$. While doing this, we choose $\dot{m}=0.05$ and $\alpha_{B}=0.01$, respectively and freely vary the other flow parameters. In the plot, solid, dotted and dashed curves illustrate the results for $\beta_{\text {in }}=6,10$ and 1000 , respectively. We find that for given $\beta_{\text {in }}, \Delta \mathcal{E}^{\max }$ increases with the increase of $a_{k}$. In general, standing shock forms at a smaller radial coordinate when $a_{k}$ is increased (Aktar, Das, \& Nandi 2015) and hence, the thermal energy content across the shock is also increased. Eventually, the accessible thermal energy likely to be dissipated at shock is also enhanced. Therefore, for a given $\beta_{\text {in }}$, we find a positive correlation between $\Delta \mathcal{E}^{\max }$ and $a_{k}$. On the other hand, as $\beta_{\text {in }}$ is reduced, synchrotron cooling turns out to be more compelling in the flow due to the increase of magnetic field strength that ultimately reduces the thermal energy content in the PSC. Thus, $\Delta \mathcal{E}^{\max }$ diminishes with the decrease of $\beta_{\text {in }}$ for fixed $a_{k}$. Finally, if the mass, spin and accretion rate of a given black hole candidate is known, the above formalism can be employed to estimate the maximum accessible energy in the PSC region and then this unbound energy could be compared with the observed radio jet kinetic power. Such a task is under progress and would be reported elsewhere.

\section{SUMMARY}

In this paper, we study the magnetized advection accretion flow around rotating black hole where viscosity and synchrotron cooling is considered as the dominant dissipation processes. We calculate the shock induced global accretion solutions and investigate the effect of dissipation parameters, such as $\dot{m}, \alpha_{B}$ and $\beta$, in deciding the formation of shock waves. The results are summarized below.

We find that accreting matter continues to harbor standing shock waves for $a_{k} \leqslant 0.8$ (see Fig. 1-5). It may be noted that we restrict the upper limit of $a_{k}$ below its maximum allowed value (i.e., $a_{k} \rightarrow 1$ ), because the adopted potential satisfactorily mimics the space-time geometry around the rotating black hole for spin parameter $a_{k} \lesssim 0.8$ (Chakrabarti \& Mondal 2006). Furthermore, we have realized that standing shocks in magnetized accretion flow are quite common and they exist for a wide range of flow parameters (see Fig. 2-5).

Next, we quantify the range of dissipation parameters that admit the formation of standing shocks in magnetized accretion flow around rotating black holes. We find that flow can sustain shock waves even when the level of dissipation is very high. More importantly, we observe that radiative cooling acts oppositely in contrast with viscous dissipation in deciding the shock parameter space (see Fig. 6). However, the effect of cooling can not be mitigated completely by viscous heating as their dependencies on the flow variables are different. Further, we find that the possibility of shock formation always decreases with the increase of dissipation strength. Subsequently, we calculate the critical accretion rate $\left(\dot{m}^{\text {cri }}\right)$ for standing shock. When accretion rate 
exceeds the critical limit, standing shock conditions are not satisfied and consequently, standing shock disappears. We find that $\dot{m}^{\text {cri }}$ strongly depends on viscosity $\left(\alpha_{B}\right)$, magnetic fields $(\beta)$ and spin of the black hole $\left(a_{k}\right)$, respectively (see Fig. 6-9). What is more is that standing shock exists in a magnetically dominated accretion flow when the accretion rate lies in general in the sub-Eddington domain $(\dot{m}<1)$ whereas for gas pressure dominated flow, shock forms even for super-Eddington accretion rate $(\dot{m}>1)$ (see Fig. 7-9).

Further, we obtain the standing shock solution for magnetized accretion flow, where the shock is considered to be dissipative by nature. The available energy dissipated at shock $(\Delta \mathcal{E})$ is usually escaped through the disc surface that is being utilized to power the jets/outflows (Le \& Becker 2004, 2005; Das, Becker, \& Le 2009). Towards this, we compute the maximum energy dissipated at shock $\left(\Delta \mathcal{E}^{\max }\right)$ and find that $\Delta \mathcal{E}^{\max }$ increases with $a_{k}$ although its dependence on $\beta_{\text {in }}$ is very much conspicuous.

Finally, we would like to mention that the present formalism is developed by adopting a simplified pseudo potential to delineate the gravitational effect around a rotating black hole. Incidentally, while studying the non-linear shock solutions, this approach allow us to avoid the mathematical complexity of general theory of relativity and at the same time it retains the salient features of space-time geometry around rotating black holes (Chakrabarti \& Mondal 2006). In this regard, although the present formalism introduces a bit of imperfections, however, we believe that the basic findings of this work will qualitatively remain unaltered due to this approximation.

\section{ACKNOWLEDGMENTS}

Authors thank the anonymous referee for useful comments and constructive suggestions.

\section{REFERENCES}

Aitken D. K., Wright C. M., Smith C. H., Roche P. F., 1993, MNRAS, 262, 456

Akizuki C., Fukue J., 2006, PASJ, 58, 469

Aktar R., Das S., Nandi A., 2015, MNRAS, 453, 3414

Aktar R., Das S., Nandi A., Sreehari H., 2017, MNRAS, 471,4806

Balbus S. A., Hawley J. F., 1991, ApJ, 376, 214

Balbus S. A., Hawley J. F., 1998, RvMP, 70, 1

Blandford R. D., Payne D. G., 1982, MNRAS, 199, 883

Blandford R. D., Znajek R. L., 1977, MNRAS, 179, 433

Becker P. A., Kazanas D., 2001, ApJ, 546, 429

Becker P. A., Das S., Le T., 2008, ApJ, 677, L93

Chakrabarti S. K., 1989, ApJ, 347, 365

Chakrabarti S. K., Molteni D., 1993, ApJ, 417, 671

Chakrabarti S., Titarchuk L. G., 1995, ApJ, 455, 623

Chakrabarti S. K., 1996a, ApJ, 464, 664

Chakrabarti S. K., 1996b, MNRAS, 283, 325

Chakrabarti S. K., Das S., 2004, MNRAS, 349, 649

Chakrabarti S. K., Mondal S., 2006, MNRAS, 369, 976

Chattopadhyay I., Chakrabarti S. K., 2000, IJMPD, 9, 717

Chattopadhyay I., Chakrabarti S. K., 2002, MNRAS, 333, 454
Chuss D. T., Davidson J. A., Dotson J. L., Dowell C. D., Hildebrand R. H., Novak G., Vaillancourt J. E., 2003, ApJ, 599, 1116

Das S., Chattopadhyay I., Chakrabarti S. K., 2001a, ApJ, 557, 983

Das S., Chattopadhyay I., Nandi A., Chakrabarti S. K., 2001b, A\&A, 379, 683

Das S., 2007, MNRAS, 376, 1659

Das S., Chakrabarti S. K., 2008, MNRAS, 389, 371

Das S., Becker P. A., Le T., 2009, ApJ, 702, 649

Das S., Chakrabarti S. K., Mondal S., 2010, MNRAS, 401, 2053

Das S., Aktar R., 2015, ASInC, 12,

Fukue J., 1987, PASJ, 39, 309

Fukue J., 1990, PASJ, 42, 793

Fukumura K., Tsuruta S., 2004, ApJ, 611, 964

Fukumura K., Takahashi M., Tsuruta S., 2007, ApJ, 657, 415

Fukumura K., Hendry D., Clark P., Tombesi F., Takahashi M., 2016, ApJ, 827, 31

Gu W.-M., Lu J.-F., 2001, ChPhL, 18, 148

Gu W.-M., Lu J.-F., 2004, ChPhL, 21, 2551

Hirose S., Krolik J. H., Stone J. M., 2006, ApJ, 640, 901

Johansen A., Levin Y., 2008, A\&A, 490, 501

Khesali A., Faghei K., 2008, MNRAS, 389, 1218

Khesali A., Faghei K., 2009, MNRAS, 398, 1361

Komissarov S. S., McKinney J. C., 2007, MNRAS, 377, L49

Kumar R., Chattopadhyay I., 2013, MNRAS, 430, 386

Le T., Becker P. A., 2004, ApJ, 617, L25

Le T., Becker P. A., 2005, ApJ, 632, 476

Lu J.-F., Gu W.-M., Yuan F., 1999, ApJ, 523, 340

Machida M., Nakamura K. E., Matsumoto R., 2006, PASJ, 58, 193

Matsumoto R., Kato S., Fukue J., Okazaki A. T., 1984, PASJ, 36, 71

Molteni D., Lanzafame G., Chakrabarti S. K., 1994, ApJ, 425,161

Mondal S., Chakrabarti S. K., 2006, MNRAS, 371, 1418

Mosallanezhad A., Abbassi S., Beiranvand N., 2014, MNRAS, 437, 3112

Mosallanezhad A., Bu D., Yuan F., 2016, MNRAS, 456, 2877

Narayan R., Yi I., 1995, ApJ, 452, 710

Nishikawa K.-I., Richardson G., Koide S., Shibata K., Ku-

doh T., Hardee P., Fishman G. J., 2005, ApJ, 625, 60

Novak G., et al., 2003, ApJ, 583, L83

Okuda T., 2014, MNRAS, 441, 2354

Okuda T., Das S., 2015, MNRAS, 453, 147

Oda H., Machida M., Nakamura K. E., Matsumoto R., 2007, PASJ, 59, 457

Oda H., Machida M., Nakamura K. E., Matsumoto R., 2010, ApJ, 712, 639

Oda H., Machida M., Nakamura K. E., Matsumoto R., Narayan R., 2012, PASJ, 64, 15

Ryu D., Chakrabarti S. K., Molteni D., 1997, ApJ, 474, 378

Samadi M., Abbassi S., Khajavi M., 2014, MNRAS, 437, 3124

Sarkar B., Das S., 2013, ASInC, 8, 143

Sarkar B., Das S., 2015, ASInC, 12,

Sarkar B., Das S., 2016, MNRAS, 461, 190

Sarkar B., Das S., 2018, JApA, 39, 3 
Sarkar B., Das S., Mandal S., 2018, MNRAS, 473, 2415

Shakura N. I., Sunyaev R. A., 1973, A\&A, 24, 337

Shapiro S. L., Teukolsky S. A., 1983, Black Holes, White Dwarfs and Neutron Stars: The Physics of Compact Objects. Wiley, New York

Singh C. B., Chakrabarti S. K., 2011, MNRAS, 410, 2414

Suková P., Janiuk A., 2015, MNRAS, 447, 1565

Suková P., Charzyński S., Janiuk A., 2017, MNRAS, 472, 4327

Takahashi M., Goto J., Fukumura K., Rilett D., Tsuruta S., 2006, ApJ, 645, 1408

Wright C. M., Aitken D. K., Smith C. H., 1993, PASAu, 10,247 